\title{
The use of capillary force for fabricating probe tips for scattering-type near-field scanning optical microscopes
}

\author{
Yoshimasa Kawata, ${ }^{\text {a) }}$ Seiji Urahama, Manambu Murakami, \\ and Futoshi Iwata \\ Department of Mechanical Engineering, Shizuoka University, Johoku, Hamamatsu 432-8561, Japan
}

(Received 7 October 2002; accepted 8 January 2003)

\begin{abstract}
We present a fabrication technique of a probe tip for scattering-type near-field microscopes. In the technique, capillary force is used to attach a metal particle at the apex of micropipettes. We can attach various kinds of particles, such as fluorescent particles, semiconductor particles, nonlinear materials, as well as metal particles. We have attached particles smaller than $180 \mathrm{~nm}$ in diameter.

(C) 2003 American Institute of Physics. [DOI: 10.1063/1.1559441]
\end{abstract}

Near-field scanning optical microscopes (NSOMs) have been widely investigated by many researchers, because they can overcome the resolution limit of an ordinary microscope. ${ }^{1-5}$ In NSOMs, a metal-coated probe tip with a small aperture at the apex is used for the illumination or the detection of lights. ${ }^{6,7}$ Recently, scattering-type NSOMs have been developed. ${ }^{8-12}$ A metal tip or a semiconductor tip without a small aperture was used for scattering light localized near the specimen surfaces. Since the metal probe greatly enhances optical electric fields at the apex of the tip, the technique is very promising with a combination of nonlinear effects, such as a two-photon process, second-harmonic generation, and coherent anti-Stokes Raman scattering. ${ }^{11,12}$

In scattering-type NSOMs, a small metal particle is preferable to scatter light, rather than a metal tip, in order to achieve high resolution, because the side of metal probes also scatters the light, as well as does the apex. Since the side of the tip scatters the propagation light, not only the localized light near the specimen surface, it decreases the resolution and the contrast of observation images.

Various techniques have been developed to implement a small metal particle for scattering light. Sugiura et al. used a laser trapping technique to scan a small metal particle. ${ }^{13,14}$ Okamoto et al. used photocatalytic deposition technique to produce a metal particle at the apex of a silicon probe tip. ${ }^{15}$ Kalkbrenner et al. made a probe with a metal particle at the apex of a sharp glass fiber by contacting the apex on the particle. $^{16}$

We present the use of capillary force to make a scattering-type probe tip. In the technique a nanometric metal particle is attached to the apex of a sharpened glass tube. The technique can easily control particle size and materials such as fluorescent particles, polystyrene particles, glass particles, semiconductor particles, etc., as well as metal particles. The probe to which a fluorescent particle is attached at the apex is also a promising tip to increase signalto-noise ratio of NSOMs, because the stray light of illumination can be greatly reduced by using interference filters or colored-glass filters.

Figure 1 shows the principle of the technique to attach a

\footnotetext{
a) Author to whom correspondence should be addressed; electronic mail kawata@eng.shizuoka.ac.jp
}

metal particle to the apex of a glass micropipette. First, we make a micropipette by sharpening a glass tube with a puller. Second, the micropipette is immersed in the water in which metal particles are dispersed. The diameter of the particle should be slightly larger than the inner diameter of the sharpened glass tube. By the capillary force in the microtube, water flows into the microtube. The water flow moves the metal particles, which are dispersed in water, toward the apex of the microtube, and one of the metal particles is trapped at the apex of the microtube. If the capillary force is not strong enough to move a particle near the tip, we can enhance the force by using a pump to suck water into the micropipette from the other side edge. When a particle is trapped at the apex of the microtube, it disturbs and stops the flow of water. As a result, it is possible to attach only one particle at the apex of the microtube.

It is useful to dither the edge of the microtube in a small region in order to select the particle which fits to the edge of the microtube. When a too-large particle, compared to the inner diameter of the microtube, is trapped, the viscosity force of the water caused by the dithering of the microtube is larger than the capillary force. Accordingly, the too-large particle is released from the apex by dithering of the microtube, and the flow of water continues until another particle is trapped to the apex.

After one particle is trapped at the apex, the micropipette is dried out. By the adhesion between the micropipette and the metal particle, we can fix a particle at the apex of the probe.

We used a fiber puller (Narishige Science Lab., PD-5)

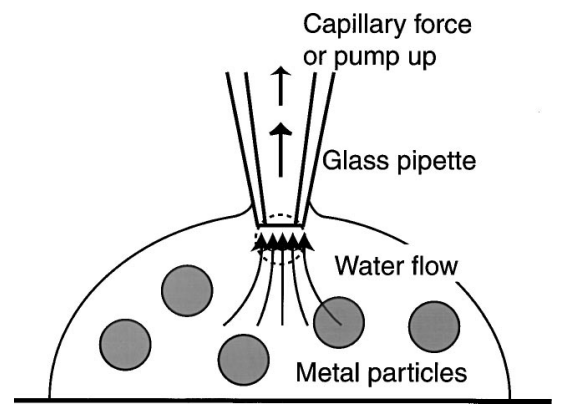

FIG. 1. Principle of attachment of a single metal particle on the apex of the micropipette using capillary force. 


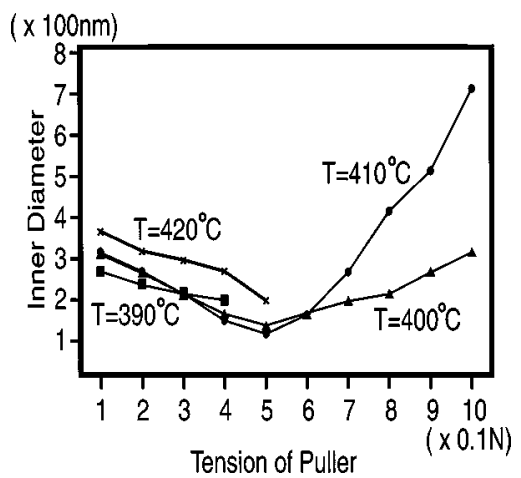

FIG. 2. The dependence of inner diameter of the micropipette on the tension of puller and on temperature.

for making microtubes. A glass tube was heated and pulled with magnetic force. Figure 2 shows the dependence of the inner diameter of the fabricated microtubes on the tension of the puller. The diameters strongly depend on the temperature of the heater and on the pulling force of the puller. The narrowest microtube was fabricated with the $0.5 \mathrm{~N}$ and $410{ }^{\circ} \mathrm{C}$ of the heater.

Figure 3 shows the edge of microtube fabricated under optimum conditions. It is recognized that the microtube with a diameter $<200 \mathrm{~nm}$ was fabricated under optimum conditions.

The fabricated microtube was immersed in water in which metal particles were dispersed. The water flowed into the microtube by the capillary force. According to the water flow, metal particles dispersed in the water were moved toward the apex of the microtube. A metal particle, the diameter of which was larger than the inner diameter of the microtube, was trapped at the apex of the microtube.

Figure 4 shows the sequential movements of a particle caused by the water flow. In order to demonstrate the particle

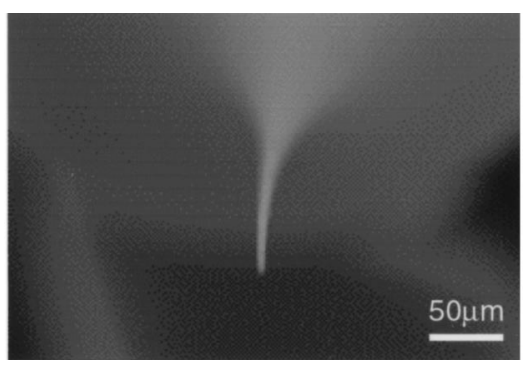

(a)

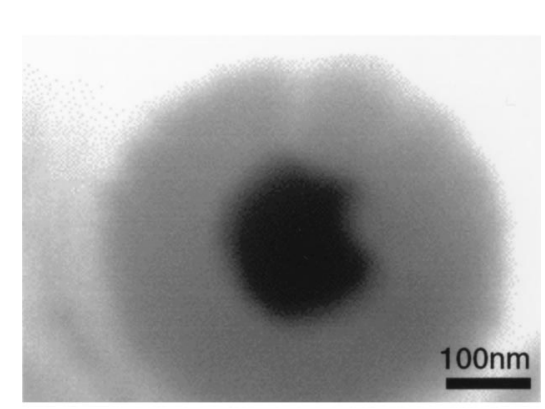

(b)

FIG. 3. Scanning electron micrographs of (a) sharpened glass tube and (b)

metal particle is attached at the apex of a microtube.
the edge of the produced micropipette.
Downloaded 10 Jul 2008 to 133.70 .80 .50 . Redistribution subject to AIP license or copyright; see http://apl.aip.org/ap

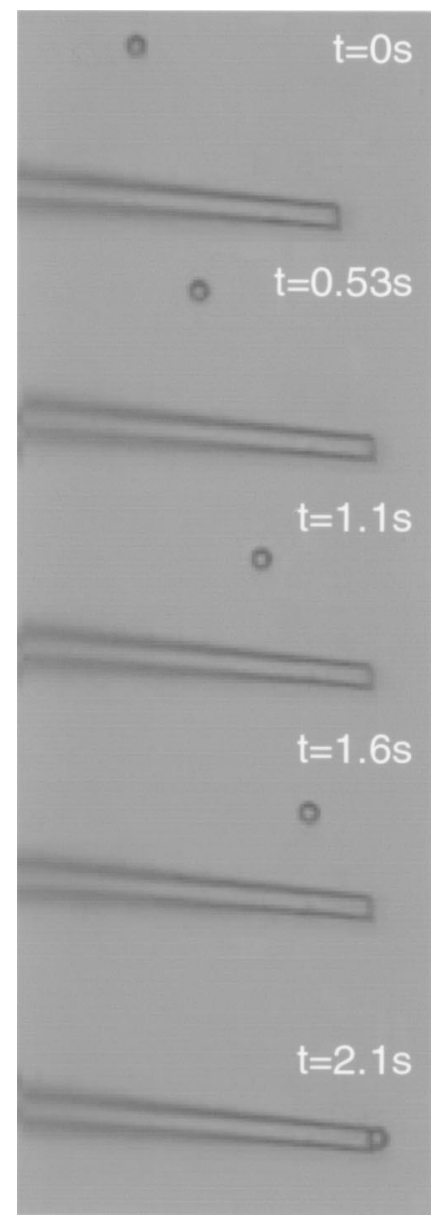

FIG. 4. Sequential movements of a particle caused by the water flow. To demonstrate, polystyrene particles of $1-\mu \mathrm{m}$ diameter were used.

movements by the capillary force, we used polystyrene particles of $1-\mu \mathrm{m}$ diameter in this experiment. We can recognize that a particle was moved toward the apex of the microtube because of the water flow.

Figure 5 shows the fabricated probe tip which has a gold particle attached at its apex. The observation image was obtained with a scanning electron microscope. The scale bar indicates $200 \mathrm{~nm}$. The diameter of the gold particle was 180 $\mathrm{nm}$. Only one particle was attached to the apex and it was fixed at the apex.

We also have tried with another type of glass tube which has a small glass rod inside of the tube in order to lead

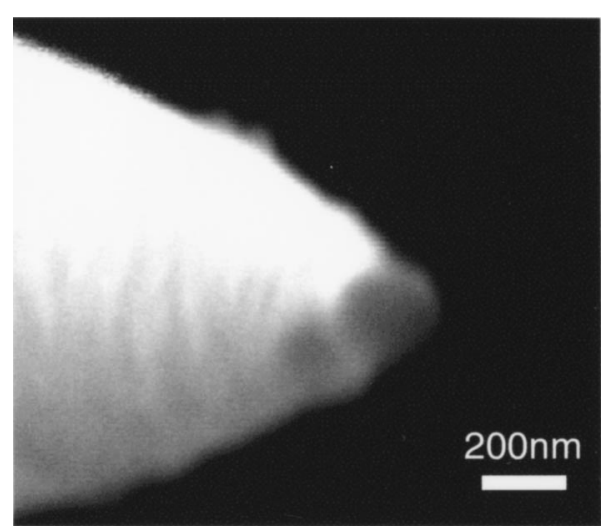

FIG. 5. Scanning electron micrograph of a fabricated probe tip. A single metal particle is attached at the apex of a microtube. 


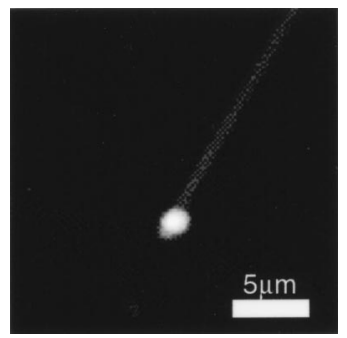

FIG. 6. Microtube image illuminated with laser light. Since the scattering efficiency of the gold particle is much higher than that of glass, the apex of the probe tip shone by the illumination.

stronger capillary flow into the microtubes. In this case, many particles were attached around the apex. The tubes created a strong flow of water and many particles moved toward the apex of the micropipettes. The trapped particle does not fit at the apex of the microtube, because the small rod inside of the tube prevents the fit of the trapped particle at the edge. Since the trapped particle did not stop the water flow, many particles were attracted to the apex and attached to the side of the microtube.

Figure 6 shows the microtube illuminated with laser light of 633-nm wavelength. Since the scattering efficiency of the gold particle is much higher than that of glass, the apex of the probe tip shone by the illumination. This is very promising to improve the resolution of scattering-type NSOMs.

In summary, we proposed technique to attach a particle at the edge of a glass micropipette by using capillary force in a microtube. The fabrication process is a kind of selforganization process and only one particle is attached at the apex, so it is very easy to fabricate probes. We have attached a metal particle of $180 \mathrm{~nm}$. The size of the particle was limited by the inner diameter of the fabricated microtube. It is possible to make a micropipette with a small aperture with a fiber puller using $\mathrm{CO}_{2}$ lasers. Apertures several tens of nanometer in size were fabricated with the systems.

Our developed technique can be applied to particles of any materials, such as polystyrene, particles containing fluorescent dye inside, semiconductor particles, crystal fragments, etc. The attachment of fluorescent particles to the probe apex is also promising in order to achieve high signalto-noise ratios in NSOMs, because the illumination light of specimens is largely eliminated with interference or colored filters. The coating of hydrophobic thin film at the side of the probe tips is also promising to prevent the adhesion of unnecessary particles on probe side surfaces.

${ }^{1}$ D. W. Pohl, W. Denk, and M. Lanz, Appl. Phys. Lett. 44, 651 (1984).

${ }^{2}$ E. Betzig, A. Lewis, A. Harootunian, M. Isaacson, and E. Kratschmer, Biophys. J. 49, 269 (1986).

${ }^{3}$ D. Courjon, K. Sarayeddine, and M. Spajer, Opt. Commun. 71, 23 (1989).

${ }^{4}$ Y. Kawata, C. Egami, O. Sugihara, N. Okamoto, M. Tsuchimori, O. Watanabe, and O. Nakamura, Opt. Commun. 161, 6 (1999).

${ }^{5}$ Y. Kawata, M. Murakami, C. Egami, O. Sugihara, N. Okamoto, M. Tsuchimori, and O. Watanabe, Appl. Phys. Lett. 78, 2247 (2001).

${ }^{6}$ H. F. Hess, E. Betzig, T. D. Harris, L. N. Pfeiffer, and K. W. West, Science 264, 1740 (1994).

${ }^{7}$ S. Mononobe, T. Saiki, T. Suzuki, S. Koshihara, and M. Ohtsu, Opt. Commun. 146, 45 (1998).

${ }^{8}$ Y. Inouye and S. Kawata, Opt. Lett. 19, 159 (1994).

${ }^{9}$ F. Zenhausern, M. P. O'Boyle, and H. K. Wickramasinghe, Appl. Phys. Lett. 65, 1623 (1994)

${ }^{10}$ F. Zenhausern, Y. Martin, and H. K. Wickramasinghe, Science 269, 1083 (1995).

${ }^{11}$ Y. Kawata, C. Xu, and W. Denk, J. Appl. Phys. 85, 1294 (1999).

${ }^{12}$ E. J. Sánchez, L. Novotny, and X. S. Xie, Phys. Rev. Lett. 82, 4014 (1999).

${ }^{13}$ T. Sugiura, T. Okada, Y. Inouye, O. Nakamura, and S. Kawata, Opt. Lett. 22, 1663 (1997).

${ }^{14}$ T. Sugiura, S. Kawata, and T. Okada, J. Microsc. 194, 291 (1999).

${ }^{15}$ T. Okamoto and I. Yamaguchi, J. Microsc. 202, 100 (2001).

${ }^{16}$ T. Kalkbrenner, M. Ramstein, J. Mlynek, and V. Sandoghdar, J. Microsc. 202, 72 (2001). 